\title{
Electron Diffraction Study of the Structure of Trimethylphosphine*
}

\author{
L. S. BARTELI, Department of Chemistry, Iowa State University, Ames, Iowa \\ AND \\ T. O. Brockwax, Department of Chemistry, University of Michigan, Ann Arbor, Michigan
}

(Received May 4, 1959)

\begin{abstract}
The structural parameters of gaseous trimethylphosphine, including standard errors, were found to be as follows: center of gravity bond distances were $r_{\mathrm{CP}}=1.846_{5} \pm 0.003 \mathrm{~A}$, and $r_{\mathrm{CI}}=1.091 \pm 0.006 \mathrm{~A}$; angles were $\angle \mathrm{C}-\mathrm{P}-\mathrm{C}=98.6 \pm 0.3^{\circ}$, and $\angle \mathrm{P}-\mathrm{C}-\mathrm{H}=110.7 \pm 0.5^{\circ}$; root-mean-square amplitudes of vibration

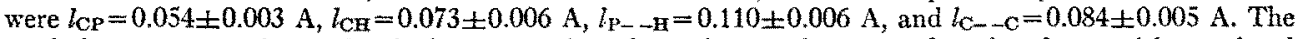
methyl groups were observed to be in staggered configurations analogous to those in ethane, with rotational barriers probably $1.5 \mathrm{kcal} / \mathrm{mole}$ or higher. A brief structural comparison with halogen derivatives and hydrides is presented.
\end{abstract}

\section{INTRODUCTION}

$\mathbf{A}$ SUBJECT of continuing speculation is the structure of trivalent compounds of group $V$ elements. The noteworthy difference in bond angles between hydrides and halides has been discussed in widely different ways. ${ }^{1}$ The methyl derivatives, being intermediate between hydrides and halides in several respects, offer useful comparisons. Since the precise structures of these compounds have not been well established, an investigation of some methyl phosphines was undertaken by modern techniques of electron diffraction. Shortly after the analysis of diffraction data for trimethyl phosphine had been carried out, a microwave determination of the structure was reported by Lide and Mann. ${ }^{2}$ This offers an excellent opportunity for comparison of the two independent structural methods.

\section{PROCEDURE}

A sample of trimethylphosphine was provided by Professor R. W. Parry. Electron diffraction patterns were recorded on Kodak Medium Lantern Slides with an apparatus described earlier, ${ }^{3}$ using $40-\mathrm{kv}$ electrons and an $r^{3}$ sector. Experimental procedures followed those previously published. ${ }^{3.4}$ Indices of resolution of 0.85 and 0.77 were obtained for the $10-\mathrm{cm}$ and $25-\mathrm{cm}$ camera data, respectively. The deviation from unity resulted principally from a uniform delocalization of the specimen throughout the diffraction chamber. Corrections for this effect were taken, conservatively, to be $2 / 3$ of the values calculated with a formula in which the deficiency of the index of resolution was as-

* This work was supported in part by the National Science Foundation.

${ }^{1}$ See for example R. S. Mulliken, J. Am. Chem. Soc. 77, 887 (1955); W. Kolos, J. Chem. Phys. 23, 1554 (1955); C. E. Mellish and J. W. Linnett, Trans. Faraday Soc. 50, 657 (1954); and articles to which the above papers refer.

${ }^{2}$ D. R. Lide, Jr., and D. E. Mann, J. Chem. Phys. 29, 914 (1958).

${ }^{3}$ L. O. Brockway and L. S. Bartell, Rev. Sci. Instr. 25, 569 (1954).

${ }^{4}$ L. S. Bartell and L. O. Brockway, J. Appl. Phys. 24, 656 (1953). sumed to be due solely to specimen delocalization. ${ }^{5}$ Corrections ranged from 0 to $0.002 \mathrm{~A}$.

The data were processed manually by a procedure closely resembling that described previously. ${ }^{6}$ The value of $b$ used in the damping function in the radial distribution function was 0.00211 . Included in the analysis were corrections for non-nuclear scattering and the failure of the Born approximation. For the latter correction the phase shifts calculated by Ibers and Hoerni $^{7}$ were used. Fourier integral termination errors were corrected with the aid of a simple approximation which, to our knowledge, has not been described before. If the Degard damping function $\exp \left(-b s^{2}\right)$ is approximated by the function $C \exp (-c s)$ at the termination of data at $s_{M}$ and beyond, it is easy to show that the termination ripple, $\Delta f_{i}$, due to peak $i$, is given approximately by

$$
\begin{aligned}
\Delta f_{i} & =\frac{f_{i} \lambda\left\{\exp \left(-\lambda^{2} s_{M}^{2} / 2\right)\right\}\left\{\beta \cos s_{M} x-x \sin s_{M} x\right\}}{(\pi / 2)^{\frac{1}{2}}\left(\beta^{2}+x^{2}\right)} \\
& =\frac{f_{i} \lambda\left\{\exp \left(-\lambda^{2} s_{M}^{2} / 2\right)\right\}\left\{\sin \left(s_{M} x+\theta\right)\right\}}{(\pi / 2)^{\frac{1}{2}}\left(\beta^{2}+x^{2}\right)^{\frac{1}{3}}}
\end{aligned}
$$

where $f_{i}$ is the maximum value of peak $i, \lambda^{2}=2 b+l^{2}$, $x=r-r_{i}, \beta=\lambda^{2} s_{M}$, and $\theta=\arctan (-\beta / x)$. This approximation is adequate for the present purpose as long as $\lambda s_{M}>\sim 2$, and $|x|<\sim r_{i}$.

Final parameters were deduced by fitting the experimental radial distribution function with Gaussian peaks, using the method of steepest ascents. ${ }^{8}$ Root-meansquare deviations between experimental and calculated curves decreased from about $0.6 \%$ to $0.3 \%$ of the maximum value of the CP peak over the interval from 1 to $3 \mathrm{~A}$. Internuclear distances are reported in terms of center of gravity parameters, $r_{\boldsymbol{v}}{ }^{9}$ Standard errors were

\footnotetext{
${ }^{5}$ L. S. Bartell, J. Appl. Phys, 32, 252 (1960).

- Bartell, Brockway, and Schwendeman, J. Chem. Phys. 23, $1854(1955)$.

7 J. A. Thers and J. A. Hoerni, Acta Cryst. 7, 405 (1954)

8 R. A. Bonham and L. S. Bartell, J. Chem. Phys. 31, 702 (1959).

- The term $r_{g}$ corresponds to the $r_{g}(0)$ of L. S. Bartell, J. Chem.
} 
calculated as described elsewhere. ${ }^{8,10}$ Standard errors quoted for bond angles correspond solely to the uncertainties in the $r_{g}$ values from which they were calculated and not to possible interpretational uncertainties arising from the operational definition of the angle.

\section{DISCUSSION OF RESULTS}

The experimental intensity function is compared in Fig. 1 with the intensity function calculated for a nearly optimum model. The radial distribution curve derived from the experimental intensity function is shown in Fig. 2. If the molecule is assumed to have $C_{3 v}$ symmetry, with methyl groups each possessing 3-fold axes approximately coincident with the $\mathrm{C}-\mathrm{P}$ bonds, the structural parameters can be completely determined from the radial distribution function. The numerical results are listed in Table I.

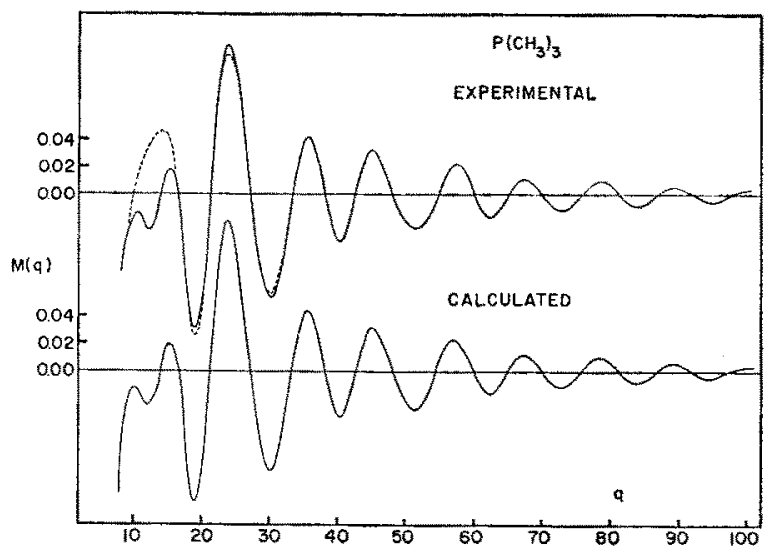

FIG. 1. Reduced intensity curves for trimethylphosphine. The solid lines represent the total scattering function $M(q)$ and the dashed line represents the nuclear scattering function $M_{\mathrm{c}}(q)$. The calculated curve has been multiplied by the experimental index of resolution.

The root-mean-square amplitudes appear to be entirely reasonable in magnitude. The distances and angles agree excellently with the microwave values of Lide and $\mathrm{Mann}^{2}$ who obtained (assuming $r_{\mathrm{CH}}=1.090 \mathrm{~A}$ ) $r_{\mathrm{CP}}=1.841 \pm 0.003 \mathrm{~A}, \quad \angle \mathrm{C}-\mathrm{P}-\mathrm{C}=99.1 \pm 0.2^{\circ}$, and $\angle \mathrm{P}-\mathrm{C}-\mathrm{H}=111.9 \pm 1^{\circ}{ }^{11}$ While none of the discrepancies between the microwave and diffraction results are significant according to Cruickshank's criterion, ${ }^{12}$ discrepancies of the observed magnitude are likely to arise because of the different operational definitions of lengths and angles involved. For example, if it is

${ }^{10}$ R. A. Bonham and L. S. Bartell, J. Am. Chem. Soc. 81, 3491 (1959).

${ }^{11}$ Lide reported the angle $\mathrm{H}-\mathrm{C}-\mathrm{H}$. Tn the present comparison it seemed preferable to avoid converting the diffraction $\angle \mathrm{P}-\mathrm{C}-\mathrm{H}$ to $\angle \mathrm{H}-\mathrm{C}-\mathrm{H}$, as the operational $\left(r_{a}\right)$ diffraction angles may not transform according to simple geometrical laws. Spectroscopic angles, which are intrinsically no more precisely defined at present, do transform simply (by hypothesis), since the individual angles are not independently defined.

${ }_{12}$ D. W.J. Cruickshank and A. P. Robertson, Acta Cryst. 6, $698(1953)$

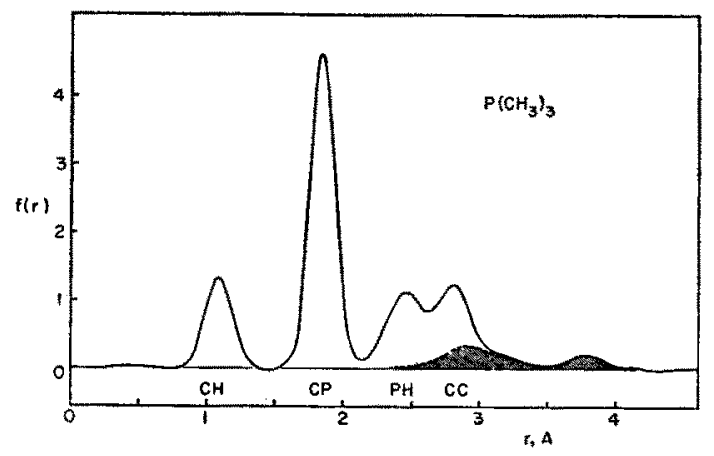

FIG. 2. Experimental radial distribution function for trimethylphosphine. The shaded area indicates the $\mathrm{C}-\mathrm{H}$ distribution characterizing the restricted rotation of the methyl groups.

considered that the $\mathrm{C}-\mathrm{P}$ bonds can be described in terms of a Morse potential function with a plausible asymmetry constant $a^{13}$ of about $1.8 \mathrm{~A}^{-1}$ and $r_{g}=$ $1.846_{5}$, the most probable $\mathrm{C}-\mathrm{P}$ distance $r_{m}$ is $1.844 \mathrm{~A}$ and $r_{j}$ is $1.839 \mathrm{~A}$ (or less if effects of nonbonded repulsions are considered). As a rule spectroscopic $r_{0}$ parameters for simple molecules appear to scatter between $r_{y}$ and $r_{e}$, generally falling closer to the latter parameter.

The present structure is also compatible with that deduced in an earlier diffraction study, ${ }^{14} r_{\mathrm{CP}}=1.87 \pm$ $0.02 \mathrm{~A}, \angle \mathrm{CPC}=100^{\circ} \pm 4^{\circ}$, to within the rather large limits of error of the earlier results. The structure of perfluorotrimethylphosphine has been investigated by Bowen, ${ }^{15}$ who obtained a $\mathrm{C}-\mathrm{P}$ bond length of $1.93_{7} \pm$ 0.017 . This distance, if correct, is remarkably longer than that in $\mathrm{P}\left(\mathrm{CH}_{3}\right)_{3}$. An analogous comparison of $\mathrm{N}\left(\mathrm{CH}_{3}\right)_{3}$ and $\mathrm{N}\left(\mathrm{CF}_{3}\right)$ reveals that the $\mathrm{C}-\mathrm{N}$ distance is shorter by perhaps several hundredths of an angstrom unit in the perfluorinated compound. ${ }^{16}$ The $\mathrm{C}-\mathrm{P}$ bond in $\mathrm{P}\left(\mathrm{CF}_{3}\right)_{3}$ merits further attention.

The diffraction $\mathrm{C}-\mathrm{H}$ distance is $0.025 \mathrm{~A}$ shorter

TABLE I. Electron diffraction results for $\mathrm{P}\left(\mathrm{CH}_{3}\right)_{3}$

\begin{tabular}{ccc}
\hline & \multicolumn{1}{c}{$r_{\sigma}, \mathrm{A}$} & \multicolumn{1}{c}{$l, \mathrm{~A}$} \\
\hline $\mathrm{C}-\mathrm{P}$ & $1.846_{6} \pm 0.003$ & $0.054 \pm 0.003$ \\
$\mathrm{C}-\mathrm{H}$ & $1.091 \pm 0.006$ & $0.073 \pm 0.006$ \\
$\mathrm{P}-\mathrm{H}$ & $2.455 \pm 0.006$ & $0.110 \pm 0.006$ \\
$\mathrm{C}-\mathrm{C}$ & $2.800 \pm 0.005$ & $0.084 \pm 0.005$ \\
$\angle \mathrm{C}-\mathrm{P}-\mathrm{C} 98.6 \pm 0.3^{\circ}$ & $\angle \mathrm{P}-\mathrm{C}-\mathrm{H} 110.7 \pm 0.5^{\circ}$ \\
Configuration of methyl groups, staggered. & \\
Barrier to rotation of methyl groups $\geqslant 1 \mathrm{kcal} /$ mole.
\end{tabular}

${ }^{13}$ L. S. Bartell, J. Chem. Phys. 23, 1219 (1955); P. M. Morse, Phys. Rev. 34, 57 (1929).

If H. D. Springall and L. O. Brockway, J. Am. Chem. Soc. 60, $996(1938)$.

${ }^{16}$ H. J. M. Bowen, Trans. Faraday Soc. 50, 783 (1954).

${ }^{16} \mathrm{~V}$. Schomaker quoted by P. W. Allen and L. E. Sutton, Acta Cryst. 3, 46 (1950); R. L. Livingston and G. Vaughan, J. Am. Chem. Soc. 78, $4866(1956)$. 


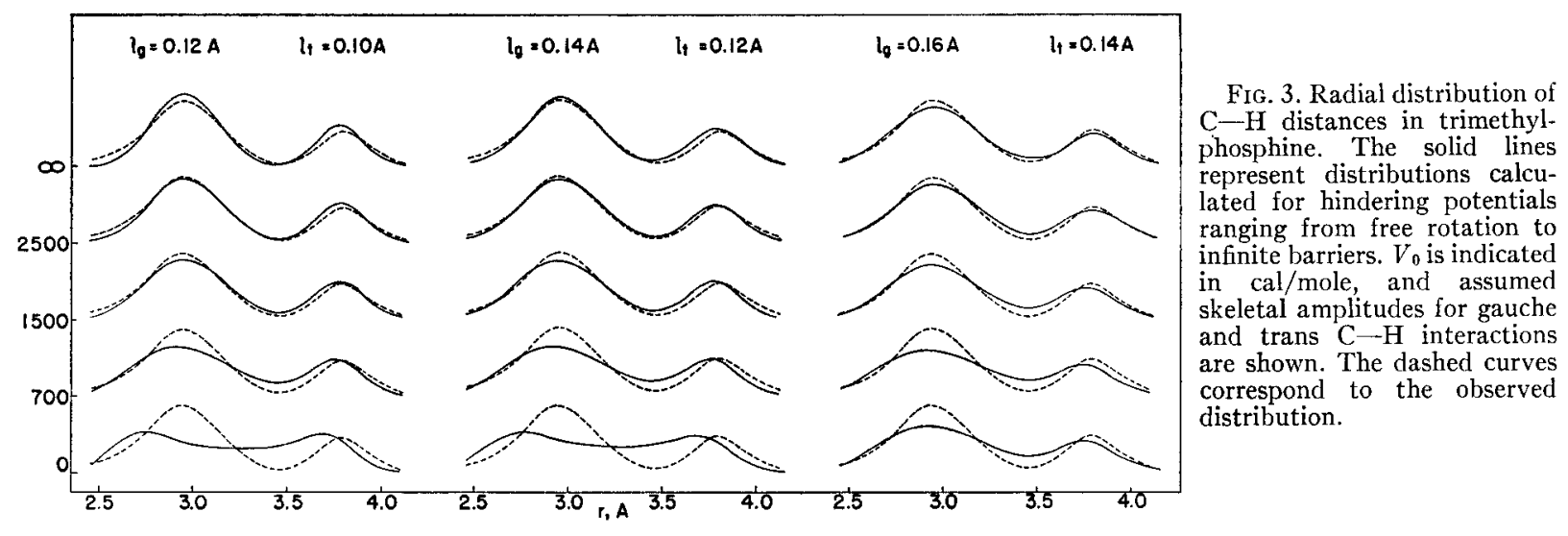

than that observed in a recent study ${ }^{17}$ of the $n$-hydrocarbons butane through heptane, and approaches in shortness the distance in ethylene. ${ }^{18}$ While this difference was also observed in mono- and dimethylphosphine ${ }^{19}$ and appears to be significant according to Cruickshank's criterion, ${ }^{12}$ there seems to be no obvious reliable explanation. Moreover, the $\mathrm{C}-\mathrm{H}$ stretching frequencies in $\mathrm{P}\left(\mathrm{CH}_{3}\right){ }_{3}{ }^{20}$ resemble those of the $n$-hydrocarbons more closely than those of ethylene. Consequently, it is unwise to regard the apparent shortness as established at present.

In addition to providing the main skeletal parameters, the diffraction data clearly indicate the orientation of the methyl groups. The groups are in a staggered configuration entirely analogous to that in ethane or isobutane. It was possible by successive approximations to make the input model in the radial distribution calculation consistent with the output data, including the range from 2.5 to $4.1 \mathrm{~A}$ which contains the information on restricted rotation of methyl groups. Plotted in Fig. 3 is this portion of the experimental radial distribution curve from which all contributions except $\mathrm{C}--\mathrm{H}$ have been subtracted. By way of comparison, theoretical curves are shown for various skeletal amplitudes of vibration and hindering potentials, assuming the azimuthal probability distribution is proportional to

$$
\exp \left[-V_{0}\left\{\cos ^{2}(3 \theta / 2)\right\} / R T\right] \text {. }
$$

Judging from amplitudes observed in other molecules, it is unlikely that the gauche $\mathrm{C}-\mathrm{H}$ (at $r \sim 2.94 \mathrm{~A}$ ) skeletal amplitude is less than $0.12 \mathrm{~A}$. It can be seen from Fig. 3 that the gauche amplitude cannot be much greater than $0.14 \mathrm{~A}$. Unfortunately it is not possible to establish the barrier independently of the skeletal amplitude, and there is at present no accurate knowledge of the amplitude. The best fit occurs with a barrier of $2.5 \mathrm{kcal}$ or greater. If the amplitude is as

17 Bonham, Bartell, and Kohl, J. Am. Chem. Soc. 81, 4765 (1959).

${ }_{18}$ L. S. Bartell and R. A. Bonham, J. Chem. Phys. 31, 400 (1959).

${ }_{19}$ L. S. Bartell, J. Chem. Phys. (to be published).

oo Rosenbaum, Rubin, and Sandberg, J. Chem. Phys. 8, 366 (1940). high as 0.14 A the diffraction data do not exclude an infinite barrier. The microwave study ${ }^{2}$ confirms the fact that the barrier is substantial, yielding the result $2.6 \pm 0.5 \mathrm{kcal} / \mathrm{mole}$. This is about $60 \%$ of the value of the analogous barrier in $\mathrm{N}\left(\mathrm{CH}_{3}\right)_{3}{ }^{21}$

The angle $\mathrm{C}-\mathrm{P}-\mathrm{C}$ is intermediate between the corresponding angle in $\mathrm{PH}_{3}$ and the halides, but is closer to the latter, about $101^{\circ},{ }^{22}$ than to the former, $93.3^{\circ} .^{22}$ Mulliken has given the most definitive review of bond angles in group $\mathrm{V}$ hydrides and halides, and invokes a variety of opposing factors governing the bond angles. ${ }^{23}$ Within his framework, the factor most appropriately differentiating phosphine from its methyl derivatives would seem to be the appreciable nonbonded repulsions between the methyl groups. Hyperconjugation could formally account for the direction of the trends but seems unlikely to be important in the ground state structures. ${ }^{24,25}$ Evidence that nonbonded repulsions can indeed account for bond angles and distances in a variety of saturated and unsaturated hydrocarbon derivatives has recently been summarized. ${ }^{25}$ It may be noted that the average $\mathrm{H}-\mathrm{C}-\mathrm{H}$ angle in the $n$-hydrocarbons butane through heptane is $7^{\circ}$ less than the average $\mathrm{C}-\mathrm{C}-\mathrm{C}$ angle, ${ }^{26}$ a difference comparable to that found between the $\mathrm{H}-\mathrm{P}-\mathrm{H}$ and $\mathrm{C}-\mathrm{P}-\mathrm{C}$ angles in the phosphines. A similar but less well documented difference has been found for amines. ${ }^{22}$

The slightly larger angles of the phosphorus halides in comparison with the methyl derivative do not result

${ }^{21}$ D. R. Lide, Jr., and D. E. Mann, J. Chem. Phys. 28, 572 (1958)

${ }^{22}$ L. E. Sutton, Tables of Interatomic Distances and Configuration in Molecules and Ions (The Chemical Society, London, 1958). ${ }^{23}$ The empirical generalizations of Mellish and Linnett (reference 1) regarding these compounds fail in the comparison of $\mathrm{PF}_{3}$ and $\mathrm{PH}_{3}$, and hence cannot be expected to apply in the case of $\mathrm{P}\left(\mathrm{CH}_{3}\right)_{3}$.

${ }_{24}$ M. J. S. Dewar and H. N. Schmeising, Tetrahedron 5, 166 (1959)

${ }_{25}$ L. S. Bartell, Federov Session on Crystallography, May 26, 1959, Leningrad, U.S.S.R. (to be published).

${ }_{26}$ Reference 17. In the calculation of the angle $\mathrm{H}--\mathrm{C}-\mathrm{H}$ from the angle $\mathrm{C}-\mathrm{C}-\mathrm{-}$ given in the reference, a slight correction has been made for the effect of the large amplitude of vibration of hydrogen atoms. 
from larger nonbonded repulsions. In halogen-substituted alkanes and silanes the angle $\mathrm{Cl}-\mathrm{Y}-\mathrm{Cl}$, as a rule, is slightly smaller, and the angle $\mathrm{F}-\mathrm{Y}-\mathrm{F}$ appreciably smaller than the angle $\mathrm{C}-\mathrm{Y}-\mathrm{C}$ in methyl analogs. ${ }^{22}$ In the phosphines the reversal of this order probably results from partial double bonding in the halides and the concomitant tendency (stronger in molecules with only three rather than four bonds) to assume a planar configuration.

\section{ACKNOWLEDGMENTS}

It is a pleasure to acknowledge the valuable assistance of Dr. R. A. Bonham in digital computations. We also wish to thank Mr. D. A. Kohl and Miss E. Bortle for aid in reading intensities and in calculations. One of us (L.S.B.) is indebted to the du Pont Company for a summer research fellowship during which the diffraction patterns were obtained.

\title{
Interactions between Ground State Oxygen Atoms and Molecules: $\mathrm{O}-\mathrm{O}$ and $\mathrm{O}_{2}-\mathrm{O}_{2}{ }^{*}$
}

\author{
Joseph T. Vanderslice, Edward A. Mason, and William G. Maisch \\ Institute for Molecular Physics, University of Maryland, College Park, Maryland
}

(Received June 22, 1959)

\begin{abstract}
Potential energy curves for $\mathrm{O}-\mathrm{O}$ interactions corresponding to the $X^{3} \Sigma_{g}{ }^{-},{ }^{1} \Delta_{g},{ }^{1} \Sigma_{u}{ }^{+},{ }^{3} \Delta_{u}, A{ }^{3} \Sigma_{u}{ }^{+}, \Sigma^{1} \Sigma_{u}^{-}$, and $B^{3} \Sigma_{a}{ }^{-}$states of $\mathrm{O}_{2}$ have been calculated from spectroscopic data by the Rydberg-Klein-Rees method. Curves for the remaining twelve states of $\mathrm{O}_{2}$ dissociating to ground state atoms have been obtained from relations derived from approximate quantum-mechanical calculations, and checked against the meager experimental information available. Two semi-independent calculations have been made, and are in good agreement with each other. The quantum-mechanical relations also lead to an approximate $\mathrm{O}_{2}-\mathrm{O}_{2}$ interaction, which is consistent with interactions derived from vibrational relaxation times and from high-temperature gas viscosity data.
\end{abstract}

\section{INTRODUCTION}

$\mathbf{T}$ HIS is the third in a series of papers dealing with the interactions among nitrogen and oxygen atoms and molecules. Such interactions are not only of importance in the calculation of transport properties of air at high temperatures, but a proper knowledge of them may also lead to a better understanding of the chemistry and physics of the upper atmosphere. Previous papers have dealt with the interactions between nitrogen atoms and molecules ${ }^{1}$ and between oxygen and nitrogen. ${ }^{2}$ The present paper deals with the calculation of the interaction energies between oxygen atoms and molecules.

The most important interactions for the calculation of transport properties are those between the ground state atoms and molecules. When two ground state oxygen atoms $\left({ }^{3} P\right)$ collide, they can follow any one of eighteen potential energy curves, ${ }^{3}$ corresponding to the spectroscopic states ${ }^{1} \Sigma_{g}{ }^{+}(2),{ }^{1} \Sigma_{u}^{-},{ }^{1} \Pi_{g},{ }^{1} \Pi_{u},{ }^{1} \Delta_{g},{ }^{3} \Sigma_{u}+(2)$, ${ }^{3} \Sigma_{g}-,{ }^{3} \Pi_{g},{ }^{3} \Pi_{u},{ }^{3} \Delta_{u},{ }^{5} \Sigma_{g}{ }^{+}(2),{ }^{5} \Sigma_{u}{ }^{-},{ }^{5} \Pi_{g},{ }^{5} \Pi_{u}$, and ${ }^{5} \Delta_{g}$. Spectroscopic data are available on the lowest six

* This research was supported in part by the National Aero nautics and Space Administration.

1 Vanderslice, Mason, and Lippincott, J. Chem. Phys. 30, 129 (1959).

2 Vanderslice, Mason, and Maisch, J. Chem. Phys. 31, 738 (1959).

${ }^{3}$ G. Herzberg, Spectra of Diatomic Molecules (D. Van Nostrand Company, Inc., Princeton, New Jersey, 1950), p. 321. bound states, $X^{3} \Sigma_{g}-,{ }^{1} \Delta_{g},{ }^{1} \Sigma_{g}+,{ }^{1} \Sigma_{u}^{-},{ }^{3} \Delta_{u}$, and $A^{3} \Sigma_{u}{ }^{+}$, as well as on the bound $B^{3} \Sigma_{u}{ }^{-}$state, which dissociates into a ground state oxygen atom $\left({ }^{3} P\right)$ and an excited atom $\left({ }^{1} D\right)$. Accurate potential energy curves for these states have been calculated by the Rydberg-KleinRees (RKR) method. ${ }^{4}$ For the other states dissociating to ground state atoms the experimental information is meager. Wilkinson and Mulliken ${ }^{5}$ suggest that the ${ }^{3} \Pi_{u}$ state predissociates the $B^{3} \Sigma_{u}-$ state at $v^{\prime}=12$ on the left-hand side of the minimum of the $B^{3} \Sigma_{u}^{-}$curve. Here $v^{\prime}$ is the vibrational quantum number of the $B^{3} \Sigma_{u}{ }^{-}$state. They also mention the possibility of a "forbidden" predissociation of the $B^{3} \boldsymbol{\Sigma}_{u}^{-}$state at $v^{\prime}=3 \frac{1}{2}$ by any of the ${ }^{5} \Sigma_{u}^{-},{ }^{1} \Pi_{u}$, or ${ }^{5} \Pi_{u}$ states. This "forbidden" predissociation would occur on the righthand side of the minimum of the $B^{3} \Sigma_{u}$ - curve.

Simple quantum-mechanical considerations have been used to determine relations among the eighteen different states of $\mathrm{O}_{2}$ dissociating to ground state atoms. Once the curves for the six bound states are known, these relations enable the "tails" of the other curves to be determined. The curves so calculated agree with the results of Wilkinson and Mulliken on the predis-

4 (a) R. Ryduerg, Z. Physik 73, 376 (1931); (b) O. Klein, Z. Physik 76, 226 (1932); (c) A. L. G. Rees, Proc. Phys. Soc. (London) 59, 998 (1947); (d) Vanderslice, Mason, Maisch, and Lippincott, J. Mol. Spectroscopy 3, 17 (1959).

5 P. G. Wilkinson and R. S. Mulliken, Astrophys. J. 125, 594 (1957). 